\title{
Decreased albumin-to-alkaline phosphatase ratio predicted poor survival of resectable gastric cancer patients
}

\author{
Yan Wang ${ }^{1 *}$, Feng Xiong ${ }^{2 \#}$, Jian Yang ${ }^{3 \#}$, Tingting Xia ${ }^{1}$, Zhenyu Jia ${ }^{1}$, Jiaqing Shen ${ }^{1}$, Chunfang Xu ${ }^{1}$, \\ Jun Feng ${ }^{2}$, Yongda Lu ${ }^{1}$ \\ ${ }^{1}$ Department of Gastroenterology, The First Affiliated Hospital of Soochow University, Suzhou, China; ${ }^{2}$ Department of Oncology, The First \\ Affiliated Hospital of Soochow University, Suzhou, China; ${ }^{3}$ Department of General Surgery, The First Affiliated Hospital of Soochow University, \\ Suzhou, China \\ Contributions: (I) Conception and design: Y Lu, J Feng; (II) Administrative support: Y Lu, J Feng; (III) Provision of study materials or patients: Y Lu, \\ J Feng, J Yang; (IV) Collection and assembly of data: Y Wang, F Xiong, T Xia, Z Jia; (V) Data analysis and interpretation: J Shen, C Xu, Y Wang, T \\ Xia; (VI) Manuscript writing: All authors; (VII) Final approval of manuscript: All authors. \\ \#These authors contributed equally to this work. \\ Correspondence to: Yongda Lu. Department of Gastroenterology Medicine, The First Affiliated Hospital of Soochow University, Suzhou 215006, \\ China. Email: ydlu@suda.edu.cn; Jun Feng. Department of Oncology, The First Affiliated Hospital of Soochow University, Suzhou, China. \\ Email: jfengsdfyy@163.com.
}

Background: The albumin-to-alkaline phosphatase ratio (AAPR) is an innovative prognostic index for various cancer patients, the clinical significance of the AAPR in patients with GC is unknown.

Methods: We retrospectively reviewed 227 resectable GC patients in our center. The Kaplan-Meier method and the Cox proportional hazards model were used to analyze the disease-free survival (DFS) and overall survival (OS). The Likelihood Ratio Test (LRT) and Akaike information criterion (AIC) were used to compare the prognostic abilities of the TNM and AAPR-TNM staging systems in DFS and OS prediction

Results: The AAPR was significantly decreased in GC patients, and the optimal cut-off value for resectable and benign gastric disease was 0.437 as determined by the receiver operating characteristic (ROC) curve. The correlation analysis revealed that decreased AAPR in GC was associated with $\mathrm{T}$ stage $(\mathrm{P}=0.004)$ and TNM stage $(\mathrm{P}=0.013)$. Decreased preoperative AAPR correlated with both unfavorable disease-free survival (DFS) and overall survival (OS). Cox regression analysis showed that the TNM stage (DFS: $\mathrm{P}=0.001$, OS: $\mathrm{P}=0.002$ ) and differential levels of AAPR (DFS: $\mathrm{P}<0.001$, OS: $\mathrm{P}<0.001$ ) were independent risk factors of DFS and OS. ROC analysis showed that the AAPR-TNM system was more superior than the TNM staging system for DFS $(\mathrm{z}=1.91, \mathrm{P}=0.028)$ and OS $(\mathrm{z}=1.937, \mathrm{P}=0.026)$ prediction. The likelihood ratio test (LRT) analysis indicated that the AAPR-TNM system had a significantly larger $\chi^{2}$ for both DFS (35.58 vs. 34.51 , $\mathrm{P}<0.001)$ and $\mathrm{OS}(32.92$ vs. 30.07, $\mathrm{P}<0.001)$, and a lower Akaike information criterion (AIC) value both for DFS $(1,032$ vs. $1,065, \mathrm{P}<0.001)$ and $\mathrm{OS}(869$ vs. $898, \mathrm{P}<0.001)$ compared to the TNM system.

Conclusions: The AAPR level significantly decreased in patients with GC, and impacted the prognosis of patients.

Keywords: Albumin-to-alkaline phosphatase ratio; gastric cancer; prognosis

Submitted Mar 31, 2021. Accepted for publication Aug 16, 2021.

doi: 10.21037/jgo-21-430

View this article at: https://dx.doi.org/10.21037/jgo-21-430 


\section{Introduction}

Gastric cancer (GC) is the second most common cancer and the third leading cause of cancer-related death in China, with an estimated 423,500 newly diagnosed patients and 298,500 deaths in 2012 (1). Curative gastrectomy, followed by adjuvant chemotherapy or chemoradiotherapy, is the most commonly used strategy for resectable GC management (2). However, local recurrence and distant metastasis are inevitable for most of these patients. A total of $70-80 \%$ of node-positive patients will experience recurrence and metastasis in 5 years, and most of them will die from GC, which leads to a low 5-year survival rate. Many instruments have been developed to predict the prognosis of GC, including histological classification, TNM staging, and even molecular classifications by The Cancer Genome Atlas (TCGA) program. However, the molecular classification method of GC is so complicated and costly that it has not been routinely used in clinical practice. Therefore, it is necessary to search for effective biomarkers for GC patients to predict prognosis and develop individualized therapies.

In the recent decades, studies have found inflammation plays critical roles in all the process of cancer, including initiation, prompting, and metastasis (3). As is well-known, Helicobacter pylori (H. pylori) infection is an important cause of gastric cancer. Cytokines, one of the most critical mediators are involved in all of the process of gastric cancer. For example, interleukin-1 (IL-1 $\beta$ ) polymorphisms was first to shown associated with an increased risk of gastric cancer, especially in $H$. pylori related, which indicates IL- $1 \beta$ is a critical participant in gastric cancer initiation (4). On the other hand, cytokines are detected in microenvironment of gastric cancer, and participate in prompting, metastasis and even response to chemotherapy. For instance, C-C motif chemokine 22 (CCL22), a chemokine recruiting the regulatory $\mathrm{T}$ cells, expressed in gastric cancer, and CCL22 positive expression shown more adverse prognosis than the negative expression (5). Moreover, patients of stage II/III gastric cancer with CCL22 positive expression benefited from 5 -fluorouracil based adjuvant chemotherapy (5).

Otherwise, systemic inflammation has been shown participating in process of tumorigenesis (6). Thus, many systemic inflammation indexes, derived from platelet, lymphocyte, and neutrophil, were used to predict the prognosis of cancers (6). For example, systemic immuneinflammation index, generated by platelet $x$ neutrophil/ lymphocyte, was shown as an independent prognostic factor for overall survival in patients with gastric cancer, and was much more better to neutrophil-to-lymphocyte rate (NLR) and platelet-to-lymphocyte rate (PLR) for predicting survival (7). Liver is the largest solid immune and metabolism organ, and the main target organ of gastric cancer metastasis (6). The status of liver may impact the prognosis of gastric cancer. The albumin-to-alkaline phosphatase ratio (AAPR) is an innovative prognostic index for cancer patients, which derived from liver function, and reflected systemic inflammation and nutrition status, and was first proposed in hepatocellular carcinoma (HCC) and nasopharyngeal carcinoma. Low AAPR has been shown to have a close relationship with poor prognosis in various malignancies, including renal cell carcinoma, lung cancer, breast cancer, and cholangiocarcinoma (8-12). However, the role of the AAPR in GC patients has not yet been studied. Therefore, it is of interest to investigate whether the AAPR can also be applied to GC patients.

Here, we seek to appraise the clinical significance of the AAPR in patients with GC and perform association analyses between their AAPR levels and clinicopathological characteristics, thereby acquiring more practical information for the risk stratification and clinical decisionmaking of GC.

We present the following article in accordance with the REMARK reporting checklist (available at https://dx.doi. org/10.21037/jgo-21-430).

\section{Methods}

\section{Patients}

We reviewed the GC patients who had accepted gastrectomy at the First Affiliated Hospital of Soochow University between July 2013 and December 2015. A total of 283 cases were enrolled, and 56 GC patients were excluded from this study for the following reasons: other concomitant malignancies $(\mathrm{n}=2)$, received neoadjuvant chemotherapy $(n=15)$, other diseases that could affect ALB and ALP levels such as liver disease, autoimmune disease, or bone disease ( $n=13)$, did not accept adjuvant chemotherapy $(\mathrm{n}=12)$, and missing data $(\mathrm{n}=14)$. Finally, 227 patients satisfied our inclusion criteria and were included for further study. All patients were treated according to the current guidelines, including the guideline of the National Comprehensive Cancer Network and the regulation of the Ministry of Health of the PRC.

The preoperative information included medical history, physical examination, blood cell count, serum biochemical 
examination, computed tomography (CT, including chest, abdomen, pelvic), and gastroscopy. Staging was re-evaluated according to the eighth edition of the TNM classification system and pathological information (13). The histological diagnosis and reclassification, according to Lauren's classification, were reviewed by 2 experienced pathologists independently. Moreover, 90 cases of gastric polyps were obtained as controls. All procedures performed in this study involving human participants were in accordance with the Declaration of Helsinki (as revised in 2013). The study was approved by the Ethics Committee of the First Affiliated Hospital of Soochow University and informed consent was taken from all the patients.

\section{Patient data}

The clinical characteristics of patients including age, gender, Eastern Cooperative Oncology Group (ECOG) performance status (PS), endoscopic findings, tumor site, personal history, history of gastrectomy, and treatment course were retrieved and reviewed from our hospital database. The AAPR value was calculated by dividing the serum ALB level by the serum ALP level.

\section{Statistical analysis}

Student's $t$ test was used to analyze serum data and the AAPR between patients and heathy controls. The chi-square test was performed to show the correlation between the AAPR and clinical data. The survival analysis was conducted using the Kaplan-Meier method and the Cox proportional hazards model. ROC curve analysis was used to determine the cut-off value of the AAPR, and the AUC was used to judge the predictive value of the AAPR, TNM stage, and AAPR-TNM. The LRT and AIC were used to compare the prognostic abilities of the TNM and AAPR-TNM staging systems in DFS and OS prediction. Statistical analyses were performed using R 3.5.3 (http://www.r-project.org) and IBM SPSS 25.0 software (SPSS Inc., Chicago, IL), and $\mathrm{P}<0.05$ was considered as statistically significant.

\section{Results}

\section{Patient demographic data}

A total of 227 GC patients were included in this study. The median age of the GC patients was 63 years old, ranging from 33 to 78 years old. Of these GC patients, there were 180 males and 57 females. We reviewed the records and reclassified the TNM stage based on the eighth edition of the TNM classification system and found that 29 patients had stage I, 123 had stage II, and 76 had stage III disease. The median follow-up time of these GC patients was 35 months, ranging from 8-63 months. There were 36 patients who did not receive adjuvant therapeutics, 98 patients accepted adjuvant therapeutics including 2 drugs, and the other 93 patients accepted adjuvant therapeutics including 3 drugs. Furthermore, 134 patients (59.0\%) survived, and 117 patients (51.5\%) did not experience tumor relapse. The 5 -year total survival rate was $61.0 \%$ for this GC patient cohort. The detailed clinicopathological and molecular characteristics, including smoking and drinking addictions, diabetes history, $\mathrm{H}$. pylori and hepatitis B infection, anemia, treatment, tumor location, Lauren's classification, vascular invasion, nerve invation, and HER2 status were recorded in Table 1.

\section{The AAPR level and optimal AAPR in GC patients}

To identify the AAPR level in GC patients, we included 90 benign gastric outpatients (gastric polyps) as controls. Albumin (ALB) decreased significantly in GC patients, and the mean ALB levels of GC patients and non-GC patients were $41.43 \pm 2.594$ and $44.02 \pm 3.951 \mathrm{~g} / \mathrm{L}$, respectively (Figure $1 A, \mathrm{P}<0.001$ ). Alkaline phosphatase (ALP) increased significantly in GC patients, and the mean ALP levels of GC patients and non-GC patients were $152.3 \pm 47.49$ and $92.23 \pm 60.03 \mathrm{U} / \mathrm{L}$, respectively (Figure $1 B, \mathrm{P}<0.001$ ). Furthermore, the AAPR level decreased significantly in GC patients, and the mean AAPR levels of GC patients and non-GC patients were $0.3139 \pm 0.2760$ and $0.5506 \pm 0.2313$, respectively (Figure $1 C, \mathrm{P}<0.001$ ). We also constructed a receiver operating characteristic (ROC) curve to determine the optimal AAPR value. When sensitivity and specificity were $57.71 \%$ and $96.56 \%$, respectively, an optimal AAPR cut-off value of 0.437 was confirmed (Figure 1D). A total of 83 patients whose AAPR was higher than or equal to 0.437 were classified as high AAPR, while the other 144 patients who had an AAPR less than 0.437 were classified as low AAPR. The ROC analysis showed that the area under the curve (AUC) of the AAPR was 0.811 [95\% confidence interval (CI): 0.764-0.858].

\section{Correlation analysis between the AAPR level and the clinicopathological characteristics of GC patients}

Since the AAPR decreased in GC patients, we further 
Table 1 Correlation analyses between clinicopathological characteristics and the albumin-to-alkaline phosphatase ratio (AAPR)

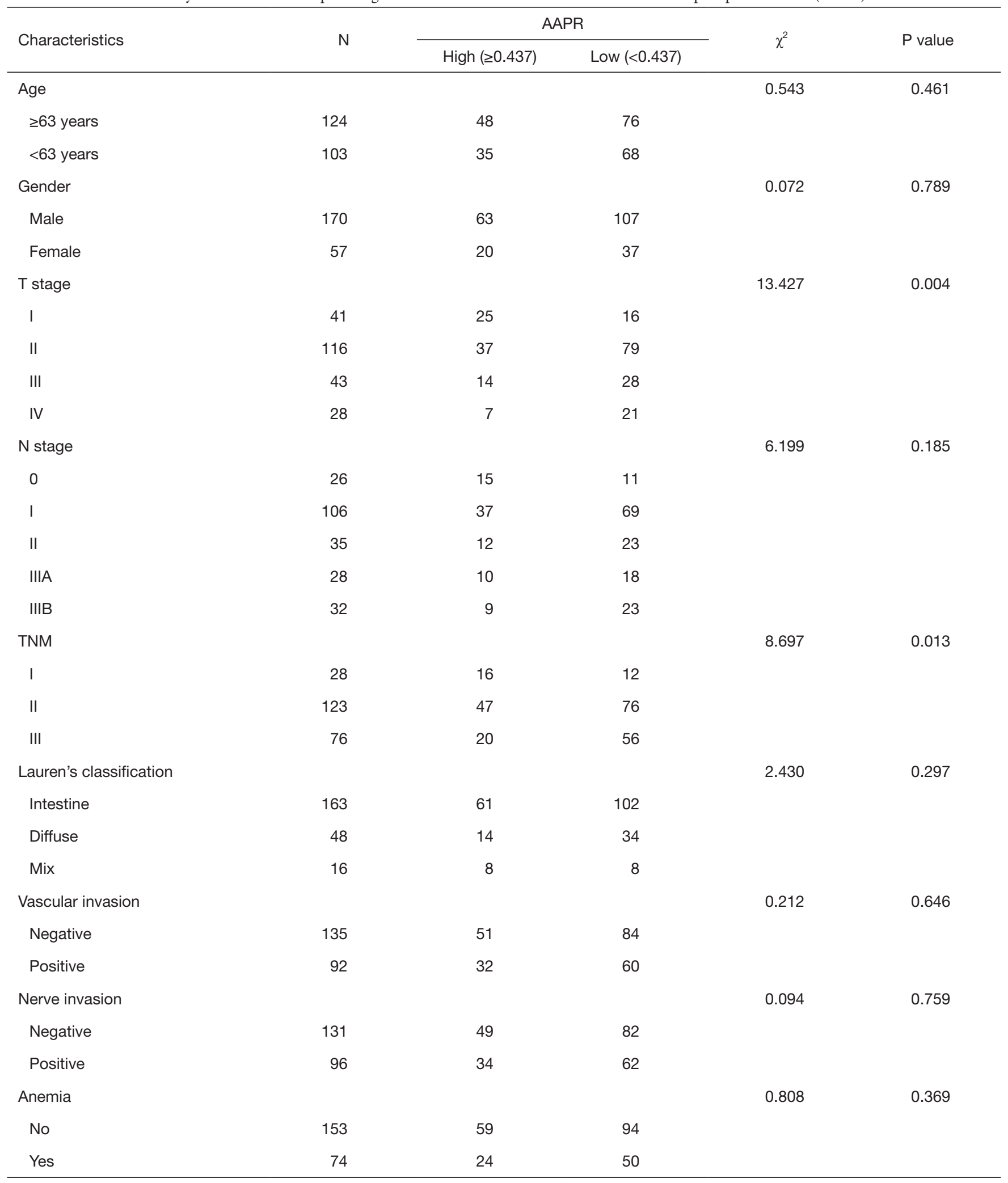

Table 1 (continued) 
Table 1 (continued)

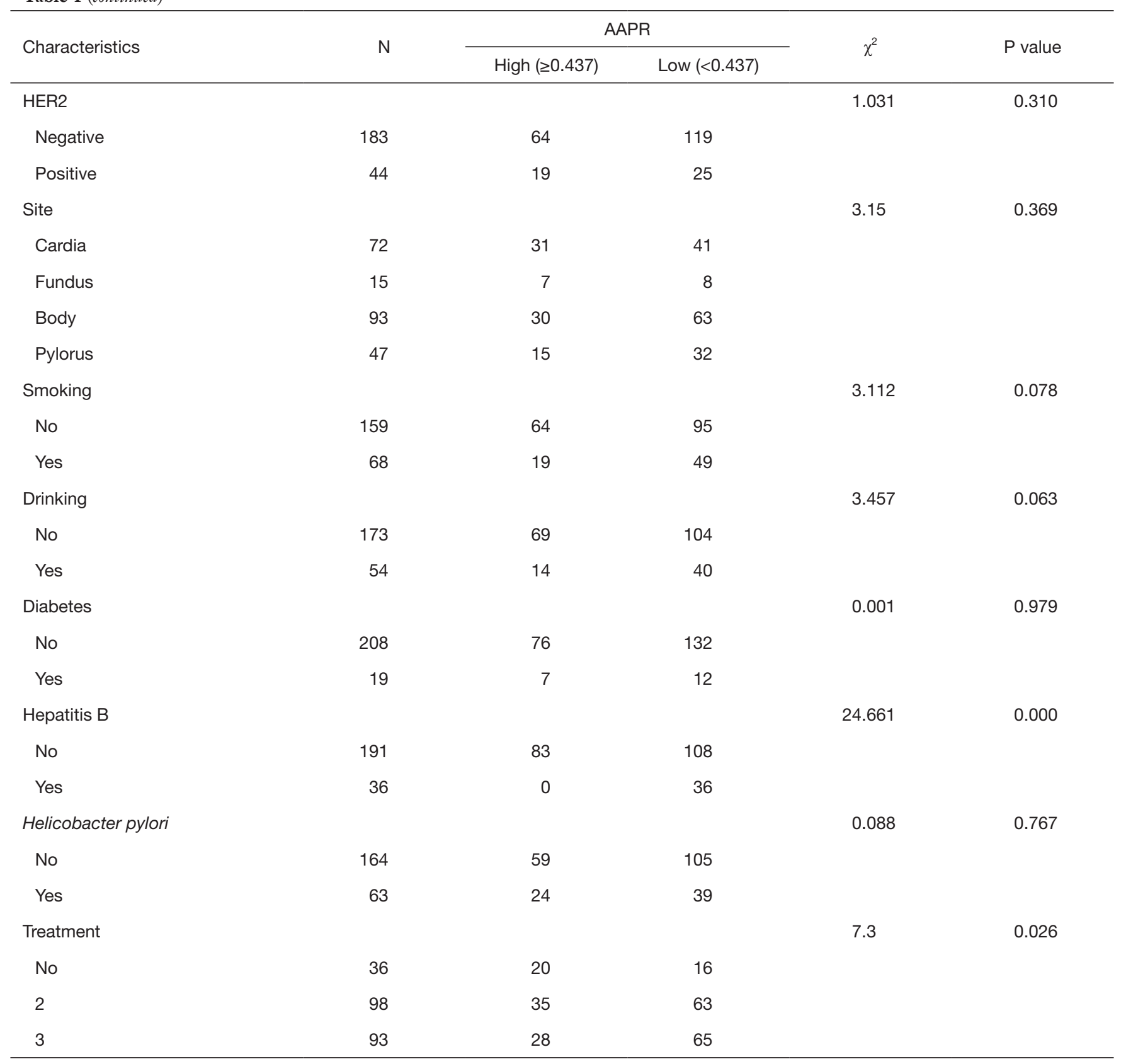

performed correlation analysis between the AAPR level and the clinicopathological characteristics of GC patients. As shown in Table 1, significant correlations were found between the AAPR level and $\mathrm{T}$ stage $(\mathrm{P}=0.004)$, TNM stage $(\mathrm{P}=0.013)$, chronic hepatitis $\mathrm{B}(\mathrm{P}=0.001)$, and adjuvant therapeutics $(\mathrm{P}=0.026)$.

\section{Survival analysis}

Of these 227 GC patients, 110 patients experienced relapse and 5 patients experienced anastomotic recurrence. The other patients were detected to have distant metastasis, such as liver [52], lung [5], lymph node [37], peritoneal [13], ovary [1] and malignant pleural [3] and peritoneal effusions [23], and 

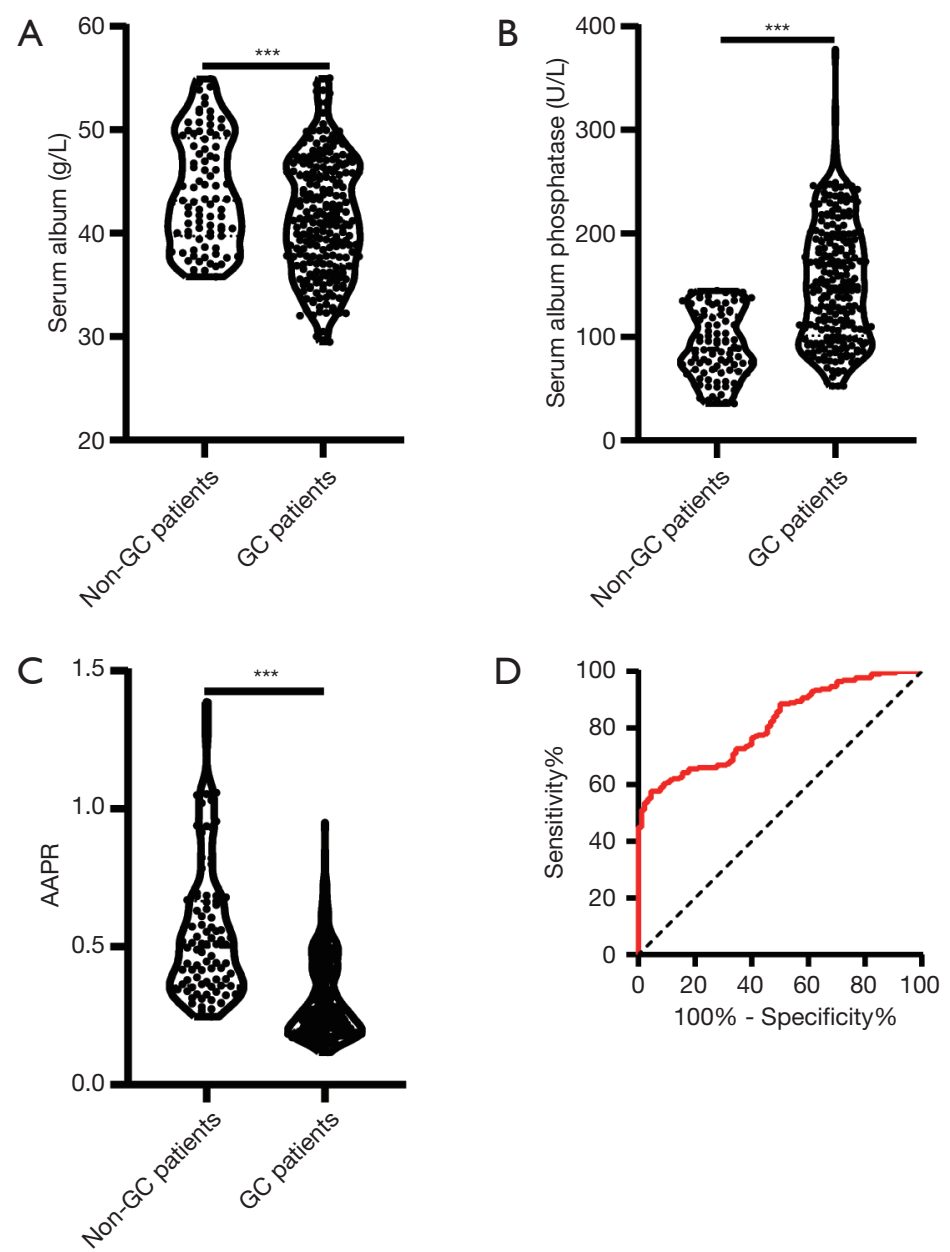

Figure 1 The albumin-to-alkaline phosphatase ratio (AAPR) decreased in patients with gastric cancer. (A) Albumin decreased in patients with gastric cancer, (B) alkaline phosphatase increased in patients with gastric cancer, (C) the AAPR decreased in patients with gastric cancer, (D) receiver operating characteristic (ROC) analysis was employed to determine the criteria for the threshold value of the AAPR for gastric cancer and benign gastric disease $(* * *, \mathrm{P}<0.001)$.

many patients were detected to have more than one distant organ involved. To further discover the potential prognostic value of the AAPR, Kaplan-Meier analysis was subsequently conducted. As shown in Figure $2 A$, the median diseasefree survival (DFS) of patients with a low AAPR level was 15 months, and the median DFS of patients with a high AAPR level was not estimated, while the accumulated DFS rates in patients with high and low AAPR levels were $72.3 \%$ and $35.4 \%$, respectively. Significantly prolonged DFS was found in patients with a high AAPR level compared to those with a low AAPR level. Then, we used the Cox proportional hazards model to assess the prognostic value of clinicopathological characteristics and the AAPR level. The univariate analysis showed that $\mathrm{T}$ stage $(\mathrm{P}=0.001), \mathrm{N}$ stage $(\mathrm{P}<0.001)$, TNM stage $(\mathrm{P}<0.001)$, vascular invasion $(\mathrm{P}=0.048)$, treatment $(\mathrm{P}<0.001)$, and AAPR $(\mathrm{P}<0.001)$ were significant factors (Table 2). Then, the multivariate analysis further confirmed that the TNM stage $(\mathrm{P}=0.013)$ and AAPR $(\mathrm{P}<0.001)$ were independent risk factors of DFS (Table 2, Figure 2B).

In this cohort, 87 patients died of cancer. Furthermore, we also conducted Kaplan-Meier analysis and Cox regression analysis to analyze the prognostic value of clinicopathological characteristics and the AAPR for overall survival (OS). The median OS of patients with a low AAPR level was 23.5 months, and the median OS of patients with a high AAPR level was not estimated (Figure 2C), while the accumulated OS rates in patients with high and low AAPR 

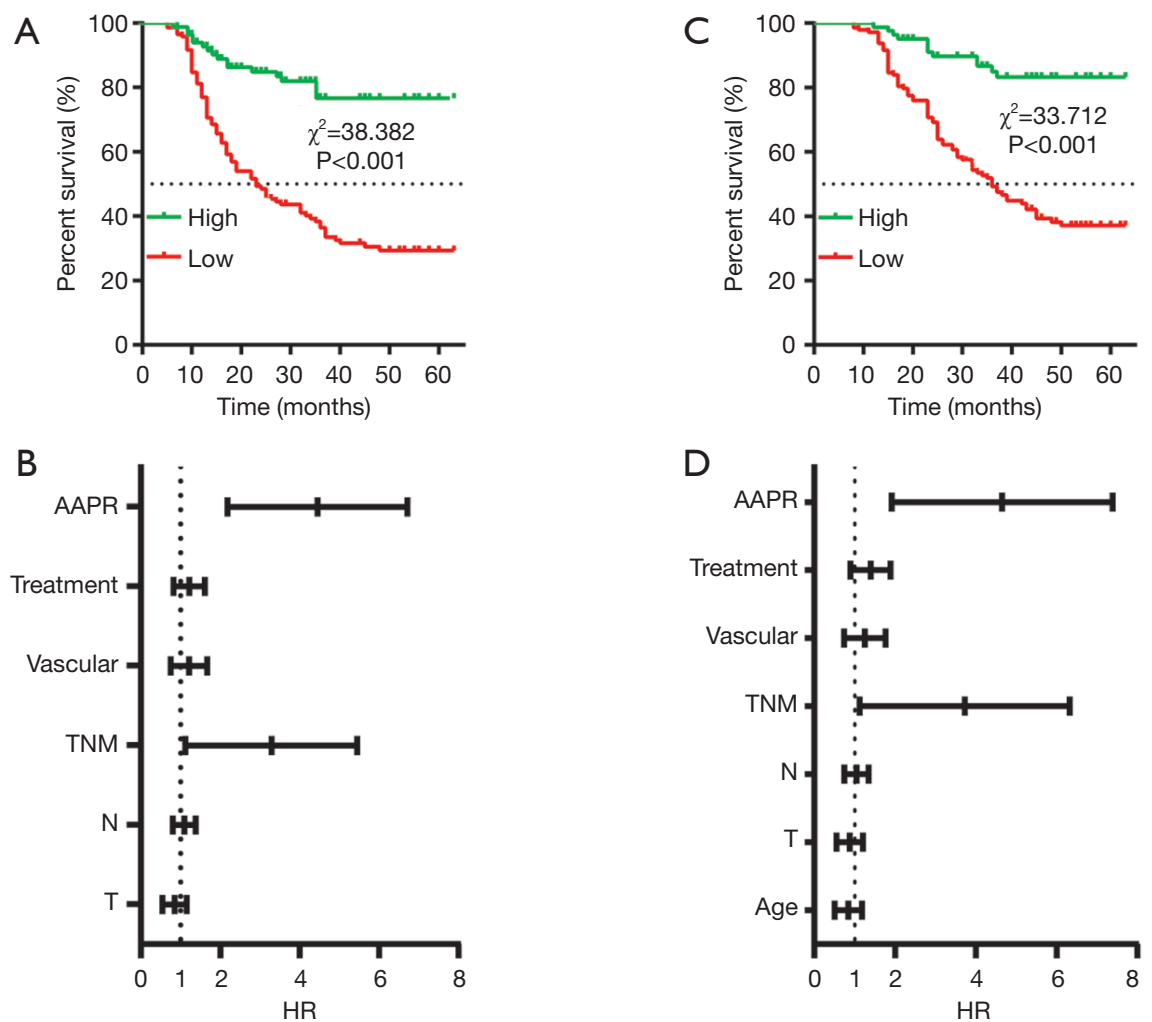

Figure 2 Survival analysis of the albumin-to-alkaline phosphatase ratio (AAPR) in gastric cancer. (A) Kaplan-Meier curve for disease-free survival (DFS) stratified by the AAPR, (B) forest plot of the multivariate analysis for DFS, (C) Kaplan-Meier curve for overall survival (OS) stratified by AAPR, (D) forest plot of the multivariate analysis for OS.

levels were $85.5 \%$ and $43.8 \%$, respectively. Significantly prolonged OS was found in patients with a high AAPR level compared to those with a low AAPR level. Moreover, the univariate analysis showed that age $(\mathrm{P}=0.044)$, $\mathrm{T}$ stage $(\mathrm{P}<0.001), \mathrm{N}$ stage $(\mathrm{P}<0.001)$, TNM stage $(\mathrm{P}<0.001)$, vascular invasion $(\mathrm{P}=0.040)$, treatment $(\mathrm{P}<0.001)$, and AAPR $(\mathrm{P}<0.001)$ were significant factors (Table 3$)$. Then, the multivariate analysis further confirmed that the TNM stage $(\mathrm{P}=0.015)$ and AAPR $(\mathrm{P}<0.001)$ were independent risk factors of OS (Table 3, Figure 2D).

\section{Comparison of the AAPR-TNM and TNM staging systems}

The TNM staging system was an independent risk factor of both DFS and OS in our previous analysis and is widely used to predict prognosis clinically. We analyzed the DFS and OS in different TNM stages stratified by the AAPR. Our results demonstrated that the AAPR could not distinguish different DFS of stage I GC patients (Figure $3 A$ ), however, the DFS in the high AAPR group outperformed those of the low AAPR group in the stage II and III subgroups (stage II, $\mathrm{P}<0.001$, Figure $3 B$; and stage III, $\mathrm{P}=0.005$ for DFS, Figure $3 C$ ). And similar results were in the OS (stage I, $\mathrm{P}>0.05$ Figure $3 D$, stage II, $\mathrm{P}<0.001$, Figure $3 E$; and stage III, $\mathrm{P}=0.008$, Figure $3 F$ ).

The 5 -year DFS rates were $85.7 \%, 56.9 \%$, and $31.6 \%$ in GC patients with stage I, II, or III disease, respectively $(\mathrm{P}<0.001$, Figure $4 A)$. To achieve more decisive outcomes, we combined the AAPR and TNM stage to create an innovative AAPR-TNM system. Briefly, we recorded stages I, II, and III as 1, 2, and 3, and recorded low and high AAPR as 0 and 1 . The AAPR-TNM score was the sum of the AAPR and TNM, which ranged from 1 to 4 . According to the innovative AAPR-TNM system, we classified the patients into four groups: grade 1, 2, 3, and 4. In this innovative AAPR-TNM staging system, the 5-year DFS rates were $93.8 \%, 89.8 \%, 52.1 \%$, and $28.6 \%$ in patients with grade $1,2,3$, or 4 disease, respectively $(\mathrm{P}<0.001$, Figure $4 B)$. ROC analysis was used to reveal the AUCs of the AAPR, TNM stage, and AAPR-TNM system, which 
Table 2 Univariate and multivariate Cox regression analyses of the prognostic factors for disease-free survival

\begin{tabular}{|c|c|c|c|c|c|c|}
\hline Characteristics & \multicolumn{3}{|c|}{ Univariate } & \multicolumn{3}{|c|}{ Multivariate } \\
\hline Age & 0.762 & $0.524-1.108$ & 0.155 & & - & \\
\hline Gender & 0.828 & $0.533-1.286$ & 0.401 & & - & \\
\hline $\mathrm{T}$ & 1.421 & $1.159-1.742$ & 0.001 & 0.815 & $0.569-1.168$ & 0.265 \\
\hline Stage & 2.690 & $1.962-3.688$ & 0.000 & 2.586 & $1.223-5.468$ & 0.013 \\
\hline Lauren's classification & 1.107 & $0.833-1.472$ & 0.483 & & - & \\
\hline Vascular invasion & 1.464 & $1.003-2.137$ & 0.048 & 1.160 & $0.790-1.703$ & 0.449 \\
\hline Nerve invasion & 1.240 & $0.852-1.805$ & 0.262 & & - & \\
\hline Site & 0.927 & $0.786-1.094$ & 0.369 & & - & \\
\hline Smoking & 1.415 & $0.960-2.087$ & 0.080 & & - & \\
\hline Drinking & 1.178 & $0.767-1.809$ & 0.455 & & - & \\
\hline Diabetes & 1.673 & $0.937-2.985$ & 0.082 & & - & \\
\hline Hepatitis B & 1.523 & $0.955-2.431$ & 0.078 & & - & \\
\hline Helicobacter pylori & 1.385 & $0.932-2.058$ & 0.107 & & - & \\
\hline Treatment & 1.684 & $1.281-2.213$ & 0.000 & 1.181 & $0.852-1.639$ & 0.318 \\
\hline AAPR & 4.407 & $2.623-7.405$ & 0.000 & 4.068 & $2.403-6.886$ & 0.000 \\
\hline
\end{tabular}

$\mathrm{HR}$, hazard ratio; $\mathrm{Cl}$, confidence interval; AAPR, albumin-to-alkaline phosphatase ratio.

were 0.705 (95\% CI: 0.636-0.773), 0.668 (95\% CI: $0.598-$ 0.737 ), and 0.760 (95\% CI: 0.698-0.822), respectively. Significance was clearly identified between the AAPRTNM and TNM for DFS prediction ( $\mathrm{z}=1.91, \mathrm{P}=0.028$, Figure $4 C$ ). The likelihood ratio test (LRT) analysis showed that the AAPR-TNM system had a markedly larger $\chi^{2}$ value (35.58 vs. 34.51, $\mathrm{P}<0.001)$ and a significantly smaller Akaike information criterion (AIC) value $(1,032$ vs. $1,065, \mathrm{P}<0.001)$ than the TNM staging system for DFS prediction. These data suggested that the AAPR-TNM system was superior to the traditional TNM system in predicting the DFS.

Moreover, the predictive value of the AAPR-TNM system was investigated for OS. In the primary TNM staging system, the 5 -year OS rates of stage I, II, and III disease were $92.9 \%, 63.4 \%$, and $39.5 \%$, respectively $(\mathrm{P}<0.001$, Figure $4 D)$. In the innovative AAPR-TNM staging system, the 5 -year OS rates of grade $1,2,3$, and 4 disease were $93.8 \%, 79.7 \%, 44.8 \%$, and $21.4 \%$, respectively
$(\mathrm{P}<0.001$, Figure 4E). Furthermore, ROC analysis revealed that the AUCs of the AAPR, TNM stage, and AAPRTNM system were 0.700 (95\% CI: 0.632-0.768), 0.676 (95\% CI: 0.606-0.746), and 0.768 (95\% CI: 0.607-0.829), respectively. The AAPR-TNM was significantly more reliable for OS prediction than the TNM staging system ( $\mathrm{z}=1.937, \mathrm{P}=0.026$, Figure $4 F)$. In addition, the LRT analysis revealed that the AAPR-TNM system had a markedly larger $\chi^{2}$ value $(32.92$ vs. $30.07, \mathrm{P}<0.001)$ and a significantly smaller AIC value (869 vs. $898, \mathrm{P}<0.001)$ than the traditional TNM staging system for OS prediction, suggesting that the AAPR-TNM system was superior to the TNM system for predicting OS.

\section{Discussion}

To definitively judge the prognosis of GC, many models have been explored, such as gene signatures, stromal- 
Table 3 Univariate and multivariate Cox regression analyses of the prognostic factors for overall survival

\begin{tabular}{|c|c|c|c|c|c|c|}
\hline Characteristics & \multicolumn{3}{|c|}{ Univariate } & \multicolumn{3}{|c|}{ Multivariate } \\
\hline Age & 0.658 & $0.437-0.989$ & 0.044 & 0.797 & $0.527-1.207$ & 0.285 \\
\hline Gender & 0.810 & $0.498-1.317$ & 0.395 & & - & \\
\hline $\mathrm{T}$ & 1.493 & $1.202-1.855$ & 0.000 & 0.833 & $0.569-1.219$ & 0.347 \\
\hline Stage & 2.964 & $2.089-4.206$ & 0.000 & 2.777 & $1.224-6.305$ & 0.015 \\
\hline Lauren's classification & 0.986 & $0.715-1.361$ & 0.934 & & - & \\
\hline Vascular invasion & 1.539 & $1.021-2.320$ & 0.040 & 1.181 & $0.778-1.792$ & 0.434 \\
\hline Nerve invasion & 1.293 & $0.860-1.942$ & 0.217 & & - & \\
\hline Site & 0.983 & $0.820-1.179$ & 0.854 & & - & \\
\hline Smoking & 1.304 & $0.852-1.994$ & 0.221 & & - & \\
\hline Drinking & 1.123 & $0.701-1.799$ & 0.629 & & - & \\
\hline Diabetes & 1.258 & $0.632-2.503$ & 0.513 & & - & \\
\hline Hepatitis B & 1.298 & $0.767-2.196$ & 0.332 & & - & \\
\hline Helicobacter pylori & 1.173 & $0.756-1.819$ & 0.477 & & - & \\
\hline Treatment & 1.892 & $1.393-2.570$ & 0.000 & 1.335 & $0.929-1.917$ & 0.118 \\
\hline AAPR & 4.966 & $2.706-9.112$ & 0.000 & 4.114 & $2.222-7.616$ & 0.000 \\
\hline
\end{tabular}

$\mathrm{HR}$, hazard ratio; $\mathrm{Cl}$, confidence interval; AAPR, albumin-to-alkaline phosphatase ratio.

immune score, immune cells in the stroma, and certain gene expression in cancer (14-17). However, various risk assessment models based on the TNM stage, histological classification, and routine blood tests are more widely accepted, as this kind of information is convenient and economical to obtain. For example, the preoperative Glasgow prognostic score (GPS) of GC patients undergoing D2 gastrectomy, a biomarker generated by combining serum $\mathrm{ALB}$ and C-reactive protein (CRP) levels, was not only associated with short-term postoperative complications, but also predicted long-term survival outcomes (18). In this study, we analyzed the probable prognostic value of a novel serum parameter, the AAPR, in 227 GC patients. We initially assessed the value of the preoperative ALB, ALP, and AAPR levels between GC patients and 90 benign gastric outpatients, and calculated the cut-off value of the AAPR. We revealed that the AAPR was a promising and effective prognostic factor for GC patients receiving curative surgery.
We also demonstrated that the AAPR-TNM system was superior to the current TNM system in outcome prediction for GC patients.

ALB, the most abundant serum protein, is produced by hepatocytes and is considered a nutritional index. It is involved in various biochemical processes, such as DNA replication, cell growth, and antioxidant effects against carcinogens (19). Also, low ALB can reflect malnutrition and impaired human immunity, including innate and acquired immunity, which leads to increased susceptibility to infection and inadequate response to cancer treatment (20). Recently, ALB has become an extensively used biomarker for predicting survival in various malignancies, including osteosarcoma, colorectal cancer, prostate cancer, HCC, and renal cell carcinoma (21-24). Mao et al. reported that a lower ALB and globulin ratio was associated with poor prognosis in patients with GC (25).

ALPs are a class of zinc-containing metalloenzymes 

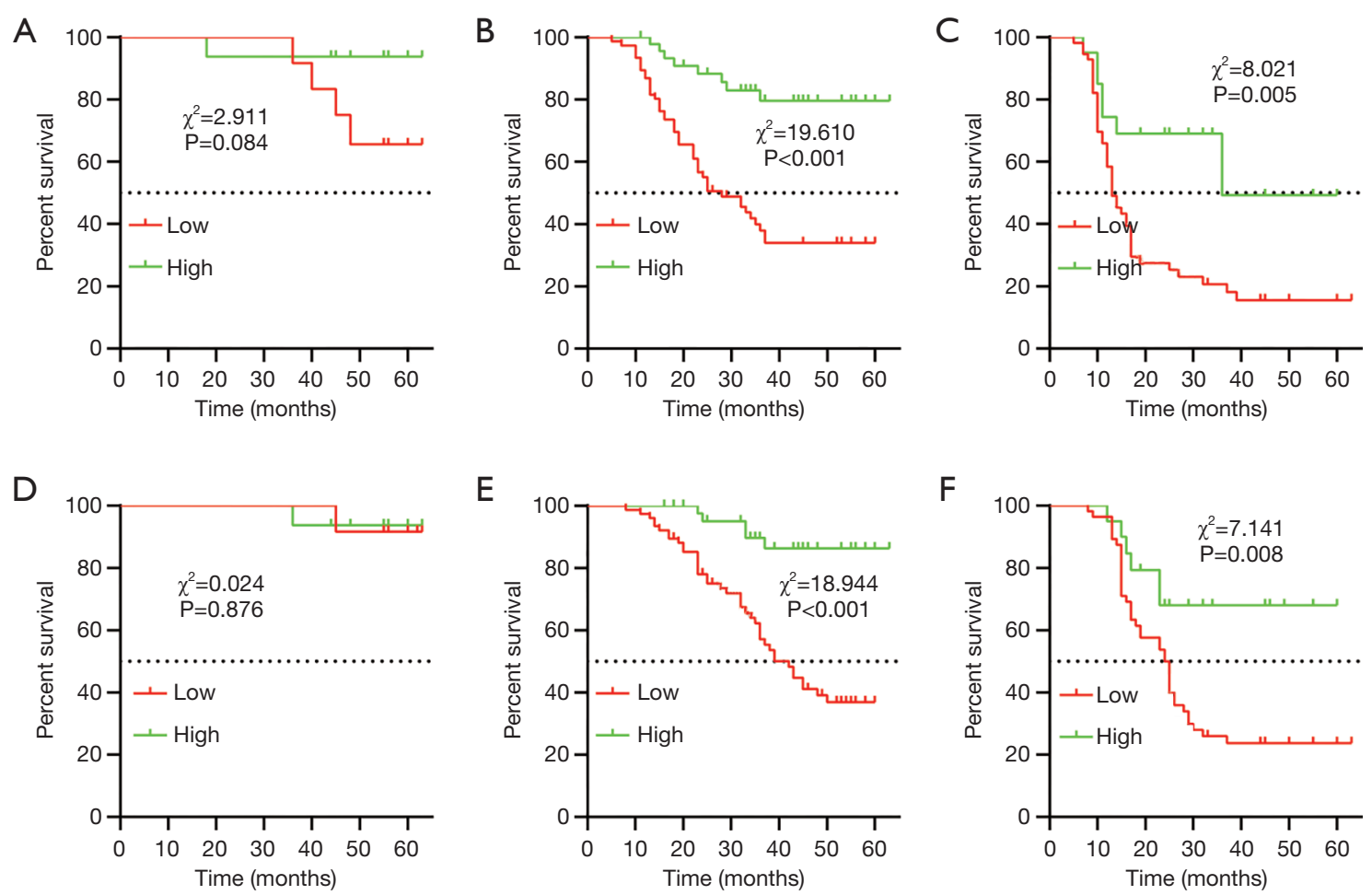

Figure 3 Kaplan-Meier curves for different TNM stages stratified by the albumin-to-alkaline phosphatase ratio (AAPR) value. KaplanMeier plots for disease-free survival (DFS) in stage I (A), stage II (B), and stage III (C). Kaplan-Meier plots for OS in stage I (D), stage II (E), and stage III (F).

which dephosphorylate the phosphate monoesters of various types of molecules at basic $\mathrm{pH}$, including nucleotides, proteins, and alkaloids. They are distributed in almost all tissues throughout the body, and are mainly concentrated in germ cells, bone, liver, intestine, kidneys, neutrophils, and placenta (26). However, aside from its role in normal skeletal mineralization, few physiological functions of ALP are known. ALP is a plasma membranebound glycoprotein which is usually confined to the cell surface, and only trace amounts of ALP are released in the sera. In adults, more than half of the activity of serum ALP results from bone ALP, and is elevated in bone, liver, and other diseases. Furthermore, ALP has been shown to be re-activated and released in some malignant diseases, such as HCC, kidney cancer, and bone metastasis $(27,28)$. Lim et al. reported that serum ALP was also associated with bone metastasis in GC (29).

It is evident that ALB and ALP affect the prognosis of cancer patients. The concept of the AAPR was found to be an effective prognostic index in resectable HCC (30). Additionally, many studies have confirmed that the AAPR has unique prognostic value and is able to better predict survival for cancer patients with both unresectable and resectable tumors $(8,9,11,12,31,32)$.

In this study, we investigated the preoperative ALB, ALP, and AAPR levels in GC patients. We found that the preoperative AAPR level decreased in GC patients, and the optimal cut-off value for GC was 0.437 . Using multivariate analysis, we found that the preoperative AAPR was an independent prognostic factor for GC patients who received D2 gastrectomy. The decreased preoperative AAPR level was associated with not only poor DFS, but also the unfavorable OS of GC patients. As is well known, the TNM staging system is a decisive index for the prognosis of GC. In this study, we also found that the TNM staging system was an independent prognostic factor for GC patients. Furthermore, we measured the effect of the AAPR in GC patients of different TNM stages separately and found that a decreased AAPR was significantly correlated with both DFS and OS in stage II and stage III disease. Then, we merged the AAPR and TNM to generate a novel AAPR-TNM system and divided the patients into 4 classes. ROC analysis 

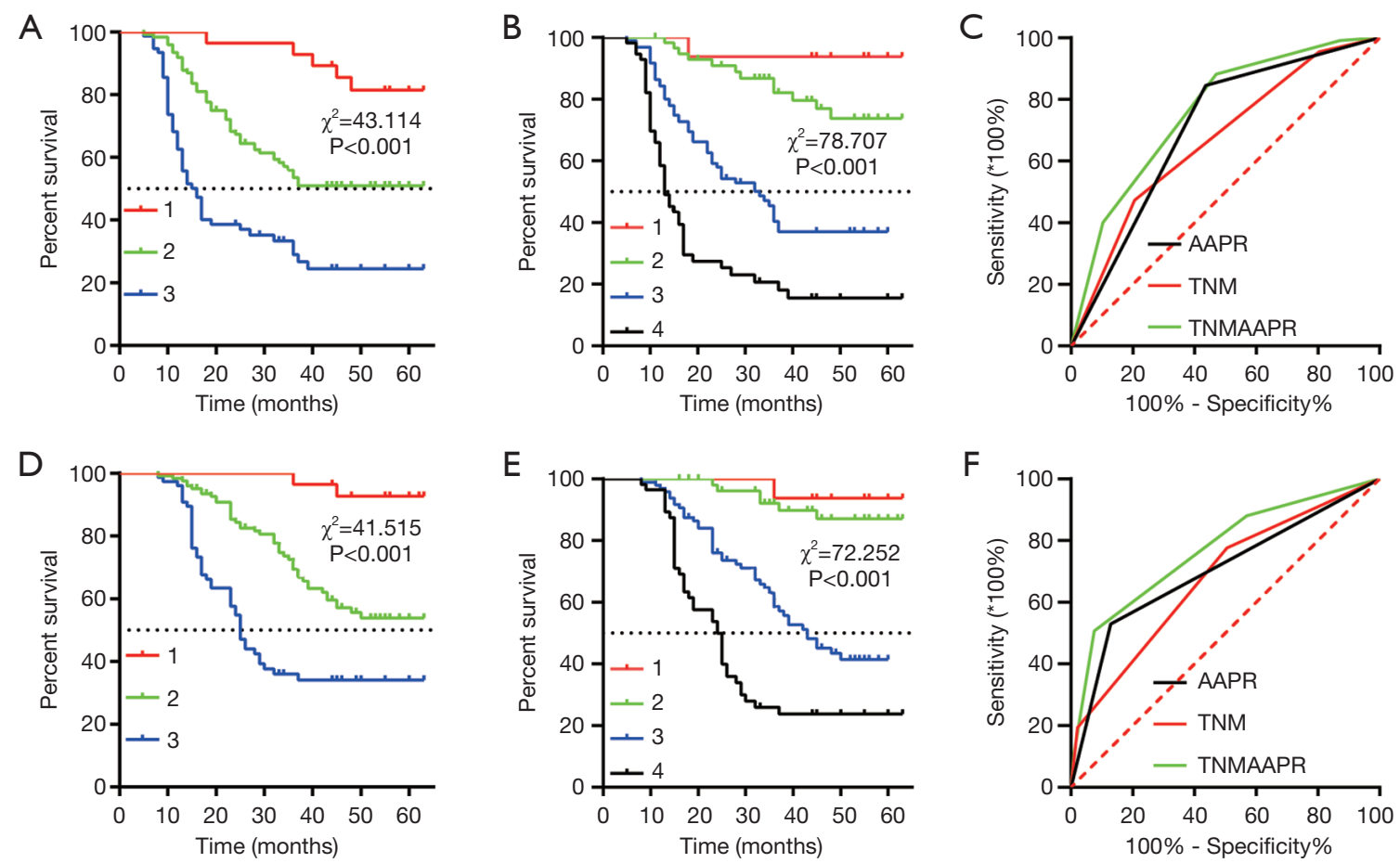

Figure 4 Kaplan-Meier curves of the TNM stage and albumin-to-alkaline phosphatase ratio (AAPR)-TNM system in gastric cancer. (A) Kaplan-Meier curve for disease-free survival (DFS) stratified by TNM stage, (B) Kaplan-Meier curve of DFS stratified by AAPR-TNM stage, (C) receiver operating characteristic (ROC) analysis of the TNM stage and the AAPR-TNM system in DFS prediction, (D) KaplanMeier curve for overall survival (OS) stratified by TNM stage, (E) Kaplan-Meier curve of OS stratified by AAPR-TNM stage, (F) ROC analysis of the TNM stage and the AAPR-TNM system in OS prediction.

showed that the AAPR-TNM system was superior to the TNM staging system for both DFS and OS prediction. Moreover, the novel AAPR-TNM classification system divided resectable GC patients into 4 independent groups, which may be more precise for predicting the prognosis of GC patients and affords more useful information for clinical decision-making. Patients in the low AAPR group are more prone to recurrence and death, and high intensity chemotherapy may deteriorate immunocompetence and accelerate tumor progression. These patients should accept enhanced nutrition, lower intensity adjuvant chemotherapy, and intensive postoperative surveillance (33). However, our study is a retrospective study, and the results should be verified in prospective multicenter studies with large sample sizes.

In conclusion, we confirmed that the AAPR significantly decreased in patients with resectable GC, and low AAPR could be used to diagnose and predict the prognosis of GC.

\section{Acknowledgments}

Funding: This work was supported by the project of Suzhou People's Livelihood Science and Technology (SYS2018037).

\section{Footnote}

Reporting Checklist: The authors have completed the REMARK reporting checklist. Available at https://dx.doi. org/10.21037/jgo-21-430

Data Sharing Statement: Available at https://dx.doi. org/10.21037/jgo-21-430

Conflicts of Interest: All authors have completed the ICMJE uniform disclosure form (available at https://dx.doi. org/10.21037/jgo-21-430). The authors have no conflicts of interest to declare. 
Ethical Statement: The authors are accountable for all aspects of the work in ensuring that questions related to the accuracy or integrity of any part of the work are appropriately investigated and resolved. All procedures performed in this study involving human participants were in accordance with the Declaration of Helsinki (as revised in 2013). The study was approved by the Ethics Committee of the First Affiliated Hospital of Soochow University and informed consent was taken from all the patients.

Open Access Statement: This is an Open Access article distributed in accordance with the Creative Commons Attribution-NonCommercial-NoDerivs 4.0 International License (CC BY-NC-ND 4.0), which permits the noncommercial replication and distribution of the article with the strict proviso that no changes or edits are made and the original work is properly cited (including links to both the formal publication through the relevant DOI and the license). See: https://creativecommons.org/licenses/by-nc-nd/4.0/.

\section{References}

1. Chen W, Zheng R, Zuo T, et al. National cancer incidence and mortality in China, 2012. Chin J Cancer Res 2016;28:1-11.

2. Hosoda K, Yamashita K, Ushiku H, et al. Prognostic relevance of FGFR2 expression in stage II/III gastric cancer with curative resection and S-1 chemotherapy. Oncol Lett 2018;15:1853-60.

3. Shalapour S, Karin M. Immunity, inflammation, and cancer: an eternal fight between good and evil. J Clin Invest 2015;125:3347-55.

4. El-Omar EM, Carrington M, Chow WH, et al. Interleukin-1 polymorphisms associated with increased risk of gastric cancer. Nature 2000;404:398-402.

5. Wu S, He H, Liu H, et al. C-C motif chemokine 22 predicts postoperative prognosis and adjuvant chemotherapeutic benefits in patients with stage II/III gastric cancer. Oncoimmunology 2018;7:e1433517.

6. McMillan D. Systemic inflammation, nutritional status and survival in patients with cancer. Curr Opin Clin Nutr Metab Care 2009;12:223-6.

7. Wang Q, Zhu D. The prognostic value of systemic immune-inflammation index (SII) in patients after radical operation for carcinoma of stomach in gastric cancer. J Gastrointest Oncol 2019;10:965-78.

8. Xia A, Chen Y, Chen J, et al. Prognostic value of the albumin-to-alkaline phosphatase ratio on urologic outcomes in patients with non-metastatic renal cell carcinoma following curative nephrectomy. J Cancer 2019;10:5494-503.

9. Zhang $\mathrm{L}$, Zhang $\mathrm{H}$, Yue $\mathrm{D}$, et al. The prognostic value of the preoperative albumin to alkaline phosphatase ratio in patients with non-small cell lung cancer after surgery. Thorac Cancer 2019;10:1581-9.

10. Xiong JP, Long JY, Xu WY, et al. Albumin-to-alkaline phosphatase ratio: A novel prognostic index of overall survival in cholangiocarcinoma patients after surgery. World J Gastrointest Oncol 2019;11:39-47.

11. Long ZQ, Hua X, Zhang WW, et al. Prognostic impact of the pretreatment albumin to alkaline phosphatase ratio for nonmetastatic breast cancer patients. Cancer Manag Res 2019;11:4809-14.

12. Li SJ, Lv WY, Du H, et al. Albumin-to-alkaline phosphatase ratio as a novel prognostic indicator for patients undergoing minimally invasive lung cancer surgery: Propensity score matching analysis using a prospective database. Int J Surg 2019;69:32-42.

13. Brierley J, Gospodarowicz MK, Wittekind C. editors. TNM Classification of Malignant Tumours. 8th ed. Oxford: Wiley Blackwell, 2017.

14. Wang H, Wu X, Chen Y. Stromal-Immune Score-Based Gene Signature: A Prognosis Stratification Tool in Gastric Cancer. Front Oncol 2019;9:1212.

15. Liu Y, Yin C, Deng MM, et al. High expression of SHMT2 is correlated with tumor progression and predicts poor prognosis in gastrointestinal tumors. Eur Rev Med Pharmacol Sci 2019;23:9379-92.

16. Liu X, Yu H, Yan C, et al. Plasmacytoid Dendritic Cells and ICOS+ Regulatory T Cells Predict Poor Prognosis in Gastric Cancer: A Pilot Study. J Cancer 2019;10:6711-5.

17. Kim SK, Kim HJ, Park JL, et al. Identification of a molecular signature of prognostic subtypes in diffuse-type gastric cancer. Gastric Cancer 2020;23:473-82.

18. Hsueh SW, Liu KH, Hung CY, et al. Significance of the Glasgow Prognostic Score in Predicting the Postoperative Outcome of Patients with Stage III Gastric Cancer. J Clin Med 2019;8:1448.

19. Arroyo V, García-Martinez R, Salvatella X. Human serum albumin, systemic inflammation, and cirrhosis. J Hepatol 2014;61:396-407.

20. Calder PC, Kew $S$. The immune system: a target for functional foods? Br J Nutr 2002;88 Suppl 2:S165-77.

21. Nazha B, Moussaly E, Zaarour M, et al. Hypoalbuminemia in colorectal cancer prognosis: Nutritional marker or inflammatory surrogate? World J Gastrointest Surg 
2015;7:370-7.

22. Yi JH, Wang D, Li ZY, et al. C-reactive protein as a prognostic factor for human osteosarcoma: a meta-analysis and literature review. PLoS One 2014;9:e94632.

23. Hiraoka A, Kumada T, Nouso K, et al. Proposed New Sub-Grouping for Intermediate-Stage Hepatocellular Carcinoma Using Albumin-Bilirubin Grade. Oncology 2016;91:153-61.

24. Chi KN, Kheoh T, Ryan CJ, et al. A prognostic index model for predicting overall survival in patients with metastatic castration-resistant prostate cancer treated with abiraterone acetate after docetaxel. Ann Oncol 2016;27:454-60.

25. Mao MJ, Wei XL, Sheng H, et al. Clinical Significance of Preoperative Albumin and Globulin Ratio in Patients with Gastric Cancer Undergoing Treatment. Biomed Res Int 2017;2017:3083267.

26. Stec B, Cheltsov A, Millán JL. Refined structures of placental alkaline phosphatase show a consistent pattern of interactions at the peripheral site. Acta Crystallogr Sect F Struct Biol Cryst Commun 2010;66:866-70.

27. Al Mamari S, Djordjevic J, Halliday JS, et al. Improvement of serum alkaline phosphatase to $<1.5$ upper limit of normal predicts better outcome and reduced risk of cholangiocarcinoma in primary sclerosing cholangitis. J Hepatol 2013;58:329-34.

Cite this article as: Wang Y, Xiong F, Yang J, Xia T, Jia Z, Shen J, Xu C, Feng J, Lu Y. Decreased albumin-to-alkaline phosphatase ratio predicted poor survival of resectable gastric cancer patients. J Gastrointest Oncol 2021;12(4):1338-1350. doi: 10.21037/jgo-21-430
28. Damera S, Raphael KL, Baird BC, et al. Serum alkaline phosphatase levels associate with elevated serum C-reactive protein in chronic kidney disease. Kidney Int 2011;79:228-33.

29. Lim SM, Kim YN, Park KH, et al. Bone alkaline phosphatase as a surrogate marker of bone metastasis in gastric cancer patients. BMC Cancer 2016;16:385.

30. Chan AW, Chan SL, Mo FK, et al. Albumin-toalkaline phosphatase ratio: a novel prognostic index for hepatocellular carcinoma. Dis Markers 2015;2015:564057.

31. Li D, Yu H, Li W. Albumin-to-alkaline phosphatase ratio at diagnosis predicts survival in patients with metastatic non-small-cell lung cancer. Onco Targets Ther 2019;12:5241-9.

32. Kim JS, Keam B, Heo DS, et al. The Prognostic Value of Albumin-to-Alkaline Phosphatase Ratio before Radical Radiotherapy in Patients with Non-metastatic Nasopharyngeal Carcinoma: A Propensity Score Matching Analysis. Cancer Res Treat 2019;51:1313-23.

33. Kanda M, Mizuno A, Tanaka C, et al. Nutritional predictors for postoperative short-term and long-term outcomes of patients with gastric cancer. Medicine (Baltimore) 2016;95:e3781.

(English Language Editor: C. Beltar) 